\title{
A prospective observational study on prescribing pattern of antimicrobial agents in the post-operative general surgery ward of a tertiary care teaching hospital
}

\author{
Velvizhy Ramalingam, Johan Pandian J.*
}

Department of Pharmacology, Mahatma Gandhi Medical College and Research Institute, Pondicherry, India

Received: 08 May 2020

Revised: 03 September 2020

Accepted: 04 September 2020

*Correspondence:

Dr. Johan Pandian J,

Email: johanpandian@gmail.com

Copyright: (C) the author(s), publisher and licensee Medip Academy. This is an open-access article distributed under the terms of the Creative Commons Attribution Non-Commercial License, which permits unrestricted non-commercial use, distribution, and reproduction in any medium, provided the original work is properly cited.

\begin{abstract}
Background: This prospective observational study was done to know the current prescription trend of antimicrobial agents in the post-operative ward. To evaluate the current pattern of antimicrobial agents in the post-operative surgical cases of a tertiary care teaching hospital.

Methods: The study was carried out in collaboration with the Department of General Surgery and Department of Pharmacology during April 2013 to March 2014. All parameters like demographic data, antimicrobials agents prescribed by surgeons, dose, frequency, duration, route, formulation, brand or generic drugs, adverse events were collected in the specially structured case proforma. Descriptive statistics was applied using SPSS version 17.0.

Results: During the study period, 513 patient case records were analysed in which males are higher than females. A total of 816 drugs were used in 484 patients during the study period. 162 were on a single drug, 190 were on two drugs, 42 were on three drugs and 90 were on fixed dose combination. Brand name of the drugs and parenteral route of administration were preferred in the study. Cephalosporins $(52.32 \%)$ and metronidazole $(34.38 \%)$ were the most commonly prescribed group of antimicrobials followed by penicillin $(0.9 \%)$, aminoglycosides $(6.58 \%)$, quinolones $(5.23 \%)$, macrolides $(0.45 \%)$ and tetracycline $(0.14 \%)$. Totally $135(27.89 \%)$ patients received drugs which are not included in the essential medicine list of WHO and $112(23.14 \%)$ patients received drugs which are not from national EML.

Conclusions: The maximum prescribed antimicrobial agent in the post-operative ward was third generation cephalosporins.
\end{abstract}

Keywords: Prescription, Cephalosporins, Aminoglycosides, Quinolones

\section{INTRODUCTION}

Infection of a wound is defined as an invasion of organisms through tissues following a breakdown of local and systemic host defences. Major wound infection occurs when a wound discharges pus and need a secondary procedure for adequate drainage which may be associated with systemic signs resulting prolongation of hospitalization, Minor wound infection presents with discharge of pus or serous fluid without excessive discomfort or systemic signs. ${ }^{1}$

Wound infection is the most usual troublesome disorder of wound healing. From the time when surgery was pioneered as a treatment modality, post-operative wound infection has been a problem. Introduction of antiseptics is considered to be an important milestone for performing surgeries in many conditions that were previously 
believed to be unacceptable due to the risk of acquiring infection in the pre-antibiotic era. ${ }^{2}$

Surgical wound infections are those infections, which are confined to the incisions and involving adjacent structures that were exposed during operation.4 Hospital acquired surgical site infection is considered as one of the major health problems throughout the world which accounts for $14-16 \%$ of the inpatient infections and has been linked with an increased risk of morbidity and mortality with huge economic impact. Roughly $77 \%$ of deaths with nosocomial infections are related to infections in post-operative wards. ${ }^{3}$

This is specifically true for the patients admitted in postoperative wards who are frequently prescribed multiple broad-spectrum antibiotics, which not only increases antibiotic resistance, but also exposes patients to unnecessary side effects along with increasing treatment costs. Nevertheless, these prescriptions are often empirical and often not rational or evidence based they lead to misuse or overuse of antimicrobial agents. ${ }^{4}$

Antimicrobials are the most commonly prescribed drugs on a worldwide basis and account for nearly $20 \%$ of all prescriptions every year. Each tertiary care hospital in the United States typically spends 1 million dollars for the purchase of antimicrobial drugs alone every year. In India, it is estimated that they account for over $50 \%$ of the total value of drugs sold. ${ }^{5}$

In most developing countries like India, it is common for antibiotics to be purchased without prescription. It has been observed that $64 \%$ of antimicrobial agents prescribed are neither indicated nor appropriate in terms of drug selection and most of them are without an appropriate bacteriological basis. In spite of wide programs on rational use of drugs and the Essential Medicine List (EML) of WHO, which are being pushed by several internal and international agencies operating in health division, irrational prescribing is still a common problem. ${ }^{6}$

The real fact is that the indiscreet use of antimicrobial agents is very common in the infirmaries. It is clear that the misuse of antimicrobials can be obviated to a greater extent in a hospital by avoiding the indiscriminate use of antimicrobial agents. However, before proposing any modification, it is mandatory to assess the existing baseline practice which can be answered with the help of a drug prescription analysis. This may therefore, help us to recognize the problems, suggest the remedial measures and encourage rational use of drugs.

Drug prescription trend can be evaluated by the analysis of clinical records of the patients in the hospital. Such cases of drug utilization studies, which are one of the tools for valuation of the health care system, provide information regarding prevalence of irrational medication in a special region.
The present work will be focused on finding out the current trends of usage of antimicrobial agents in the post-operative ward of tertiary care teaching hospital.

To evaluate the current pattern of prescribing of antimicrobial agents in the post-operative surgical cases of a tertiary care teaching hospital. To analyze the rationality of usage of antimicrobial agents in the postoperative general surgery cases. To assess the incidence of adverse drug reactions to antimicrobial agents in the post-operative general surgery cases. To analyse the resistance pattern of culture isolates to antimicrobial agents in the post-operative general surgery cases. To assess the rationale for changing over from one antimicrobial agent to another.

\section{METHODS}

This was a prospective observational study, which was conducted at the Mahatma Gandhi Medical College and Research Institute Hospital. Study duration was April 2013 to March 2014.

The population of this study was patients receiving aminoglycoside treatment, GM or AM in surgery ward with basal S. Cr level. A simple random sample of 100 patients was taken from surgery ward.

\section{Inclusion criteria}

All patients admitted in post-operative general surgery ward and antimicrobials prescribed post-operatively were only considered.

\section{Exclusion criteria}

Patient having acute or chronic renal disease with relatively less renal functions and who are undergoing haemodialysis and pregnant and lactating women

Patients who were admitted in the post-operative ward of general surgery during April 2013 to March 2014 were taken.

The prescriptions of these patients were reviewed and the details were collected at the time of their admission in post-operative ward. The following study parameters were taken down in detail in the proforma: Demographic data, name, age, sex, occupation, income and address were noted in the proforma, drugs prescribed by surgeons, different group and type of antimicrobial agents prescribed in the case records were noted down in detail, dose, frequency, duration, route, formulation, brand or generic drugs. Whether generic or brand names of the drugs were prescribed in the case records were noted, Adverse events due to use of antimicrobial drugs if recorded in case sheet. 


\section{Data collection}

All data were entered into a Data Collection Proforma Sheet (Appendix 1) and were entered into Excel (MS Excel 2010).

\section{Statistical methods}

Statistical Product and Service Solutions (SPSS) version 17 was used for statistical analysis. Descriptive statistics were applied to the data. The results were represented in the form of tables, pie charts and bar diagrams.

\section{RESULTS}

The study was carried out by the department of Pharmacology in collaboration with the department of Surgery for a period of one year (April 2013-March 2014). During the study period, 513 patient case records were analysed. Out of $513,289(56.34 \%)$ were male patients and $224(43.66 \%)$ were females.

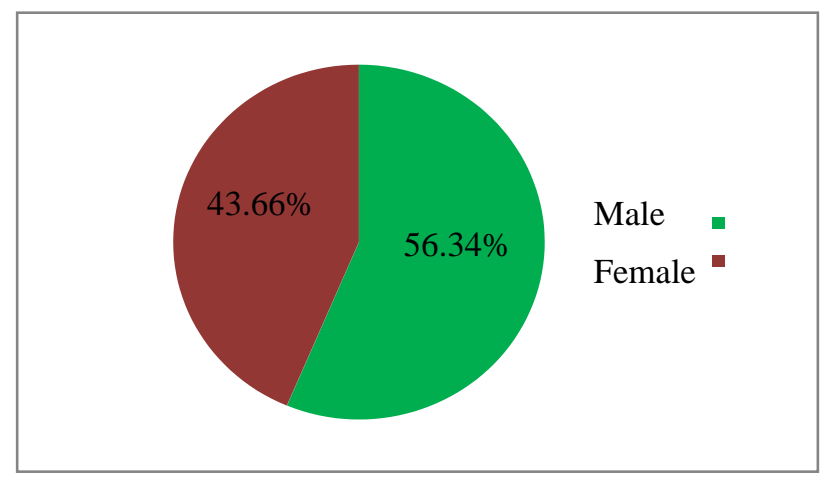

Figure 1: Gender distribution of the patients.

Age of the patients was ranging from 14 years to 80 years and the average age group of the patients was found to be 44.53 (Figure 2).

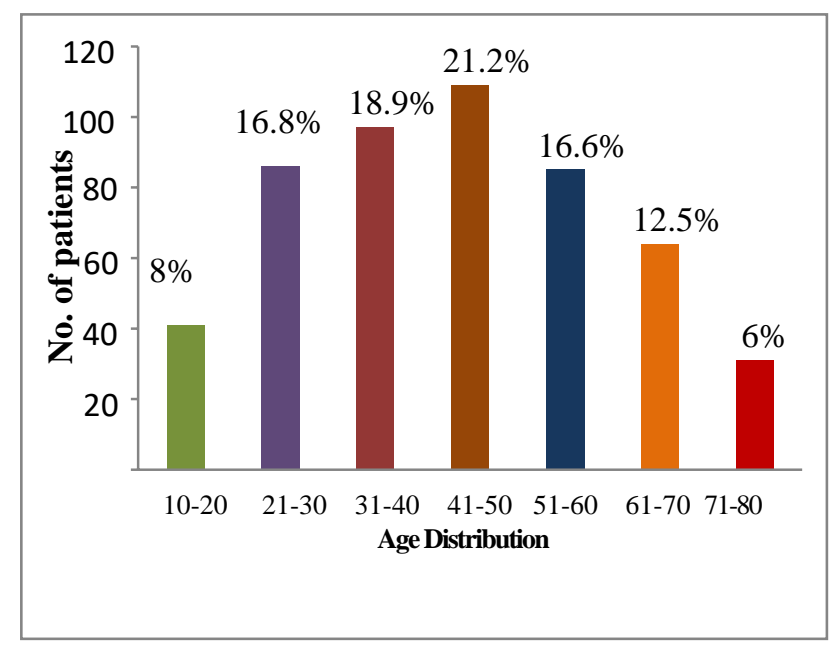

Among 513 patients, 446 were from Tamilnadu and 67 were from Puducherry. Major sample size represents Tamilnadu population and only a small sample represents Puducherry.

The most frequent diagnosis which warranted surgery was hernia, hydrocele, abscess, appendicitis, fistula, hemorrhoids, diabetic foot, necrotising fasciitis, hollow viscus perforation and fourniers gangrene.

Maximum number of drugs prescribed for a patient was 14. The average number of drugs received by patients in the present study was 9.54 .

Minimum duration of antimicrobials received by the patients was one day and the maximum duration was seventeen days. The average duration was found to be 4.09 days.

When antimicrobials received out of 513 patients studied, 29 were not prescribed any antibiotics during their stay in the post-operative ward. Remaining 484 were prescribed one or more antibiotics. A total of 816 drugs were used in 484 patients during the study period (Figure 3 ).

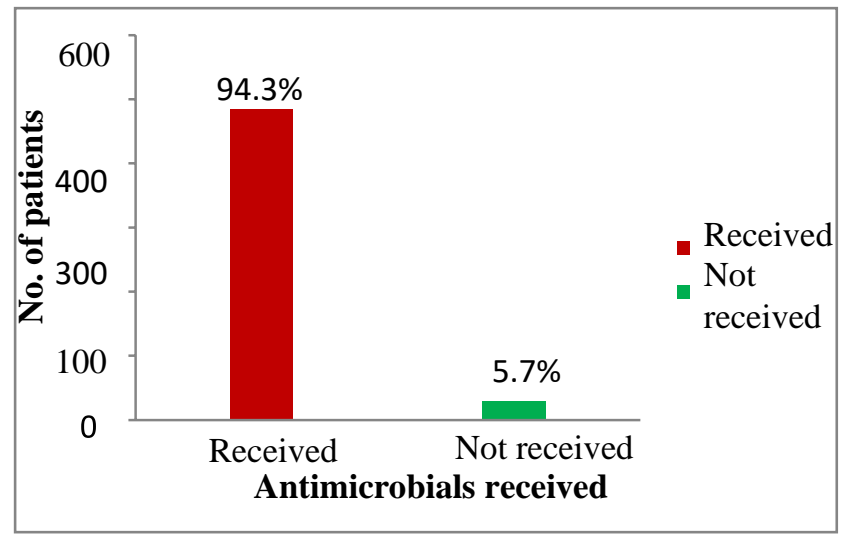

Figure 3: No. of patients received antimicrobials

\section{Types of therapy}

Where, among the 484 patients, 162 were on a single drug, 190 were on two drugs, 42 were on three drugs and 90 were on fixed dose combinations which mention in (Figure 4).

\section{Monotherapy}

In monotherapy, one hundred and fifty-one patients received cephalosporins, the most common being injection cefotaxime, seven patients received ciprofloxacin and four patients received penicillin (Table 1).

Figure 2: Age distribution of the patients. 


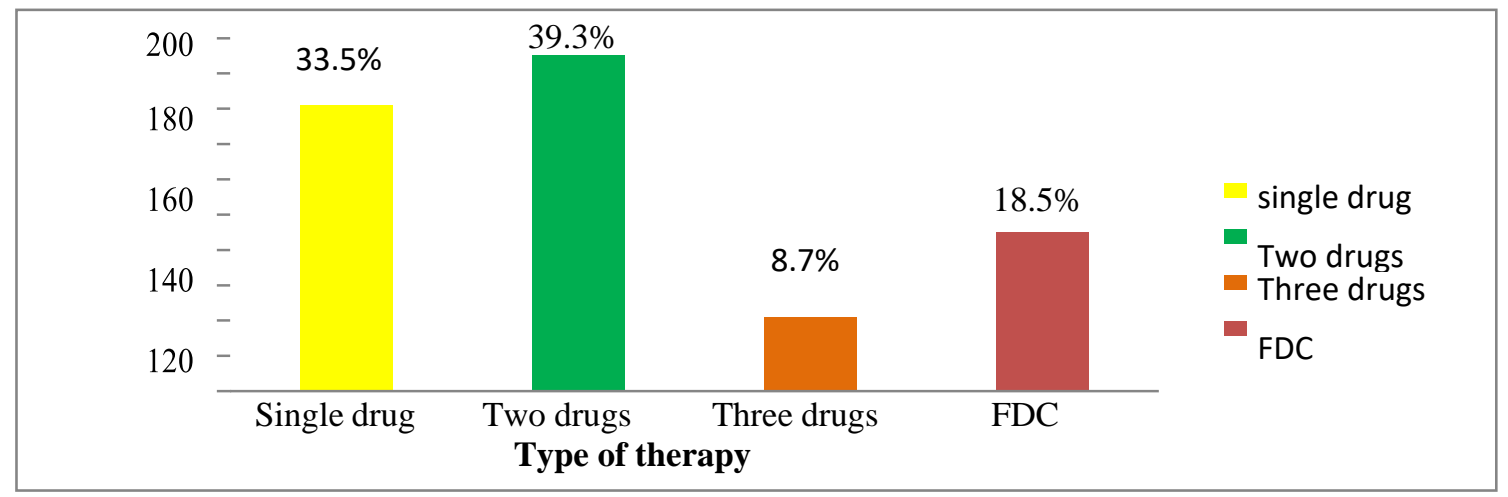

Figure 4: Type of therapy given

Table 1: No. of patients received single drug $(n=162)$.

\begin{tabular}{|lll|}
\hline Drugs & No of patients & $\%$ \\
\hline Inj. Cefotaxime & 92 & 56.8 \\
\hline Inj. Cefuroxime & 35 & 21.6 \\
\hline Inj. Ceftriaxone & 9 & 5.6 \\
\hline Inj. Ciprofloxacin & 5 & 3.1 \\
\hline Inj. Cefazolin & 3 & 1.9 \\
\hline Inj. Amoxicillin & 2 & 1.2 \\
\hline Tab. Cefixime & 12 & 7.4 \\
\hline Tab. Ciprofloxacin & 2 & 1.2 \\
\hline Tab. Penicillin G & 2 & 1.2 \\
\hline
\end{tabular}

\section{Two drugs}

A total of one hundred and ninety patients received 2 drugs. Among that 153 cases received cephalosporins with metronidazole, 26 cases received flouroquinolones with metronidazole, 4 cases received penicillins with metronidazole, 2 cases received macrolides with metronid azole and another 2 cases received fluroquinolones with aminoglycosides (Table 2).

\section{Three drugs}

Out of 42 cases, 39 patients received cephalosporins with aminoglycoside and metronidazole, 1 patient received two drugs from the cephalosporin group along with metronidazole, 1 patient received cephalosporin with metronidazole and tetracycline and another 1 patient received cephalosporin with metronidazole and macrolide (Table 3).

\section{Route of administration}

Out of 484 patients who received antimicrobial agents 438 were administered in parenteral form (intravenously), 42 patients were given orally in the form of a capsule or tablet and remaining 4 received both parenteral and oral form (Figure 5).

\section{Frequency of drugs}

In study out of 816 drugs used, 271 drugs were administered three times a day, 487 drugs were given two times a day and 58 drugs were given once a day dosing (Figure 6).

Table 2: No. of patients received two drugs $(n=190)$.

\begin{tabular}{|lll|}
\hline Drugs & $\begin{array}{l}\text { No of } \\
\text { patients }\end{array}$ & $\%$ \\
\hline $\begin{array}{l}\text { Inj. Cefotaxime + } \\
\text { inj. Metronidazole }\end{array}$ & 137 & 72.1 \\
\hline $\begin{array}{l}\text { Inj. Ciprofloxacin + } \\
\text { inj. Metronidazole }\end{array}$ & 21 & 11 \\
\hline $\begin{array}{l}\text { Inj. Ceftriaxone + } \\
\text { inj. Metronidazole }\end{array}$ & 6 & 3.2 \\
\hline $\begin{array}{l}\text { Inj. Cloxacillin+ } \\
\text { inj. Metronidazole }\end{array}$ & 4 & 2.1 \\
\hline $\begin{array}{l}\text { Inj. Cefuroxime+ } \\
\text { inj. Metronidazole }\end{array}$ & 5 & 2.6 \\
\hline $\begin{array}{l}\text { Inj. Cefotaxime + } \\
\text { inj. Gentamicin }\end{array}$ & 3 & 1.6 \\
\hline $\begin{array}{l}\text { Inj. Ciprofloxacin }+ \\
\text { inj. Amikacin }\end{array}$ & 2 & 1.1 \\
\hline $\begin{array}{l}\text { T. Cefixime+ } \\
\text { T. Metronidazole }\end{array}$ & 5 & 2.6 \\
\hline $\begin{array}{l}\text { T. Ciprofloxacin+ } \\
\text { T. Metronidazole }\end{array}$ & 5 & 2.6 \\
\hline $\begin{array}{l}\text { Inj. Metronidazole+ } \\
\text { T. Azithromycin }\end{array}$ & 2 & 1.1 \\
\hline
\end{tabular}

Table 3: No. of patients received three drugs $(n=42)$.

\begin{tabular}{|llc|}
\hline Drugs & $\begin{array}{l}\text { No of } \\
\text { patients }\end{array}$ & $\%$ \\
\hline $\begin{array}{l}\text { Inj.Cefotaxime+inj.amika } \\
\text { cin+inj. Metronidazole }\end{array}$ & 20 & 47.6 \\
\hline $\begin{array}{l}\text { Inj.Cefotaxime+inj.Genta } \\
\text { micin+inj.Metronidazole }\end{array}$ & 19 & 45.2 \\
\hline $\begin{array}{l}\text { Inj.Cefotaxime+inj.Cefuro } \\
\text { xime+inj.Metronidazole }\end{array}$ & 1 & 2.4 \\
\hline $\begin{array}{l}\text { Inj. Cefotaxime+inj. } \\
\text { Metronidazole+C. Doxy }\end{array}$ & 1 & 2.4 \\
\hline $\begin{array}{l}\text { Inj.Ceftriaxone+inj.Metro } \\
\text { nidazole+T.Erythromycin }\end{array}$ & 1 & 2.4 \\
\hline
\end{tabular}




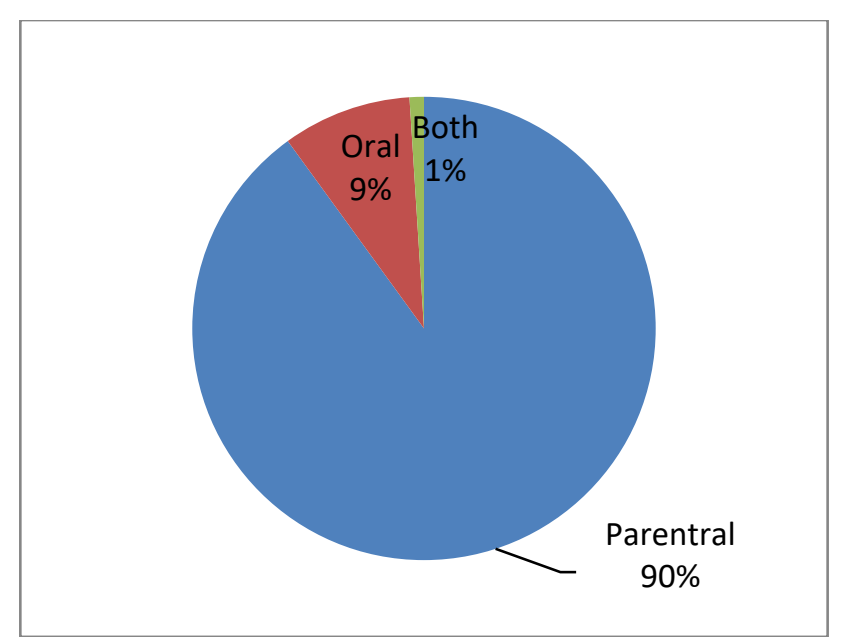

Figure 5: Route of drug administration.

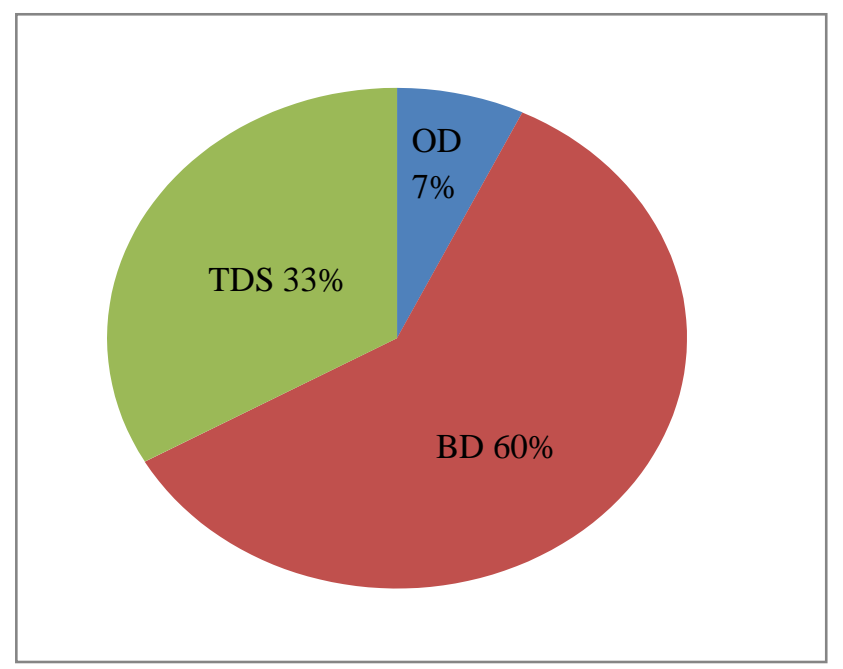

Figure 6: Frequency of drugs.

Indication for antimicrobials

816 antimicrobials were prescribed for 484 patients and the drugs were categorized based upon the cause for utilization and their percentage was found to be for prophylaxis- (296) $61.16 \%$ For treatment- (188) $38.84 \%$ (Figure 7).

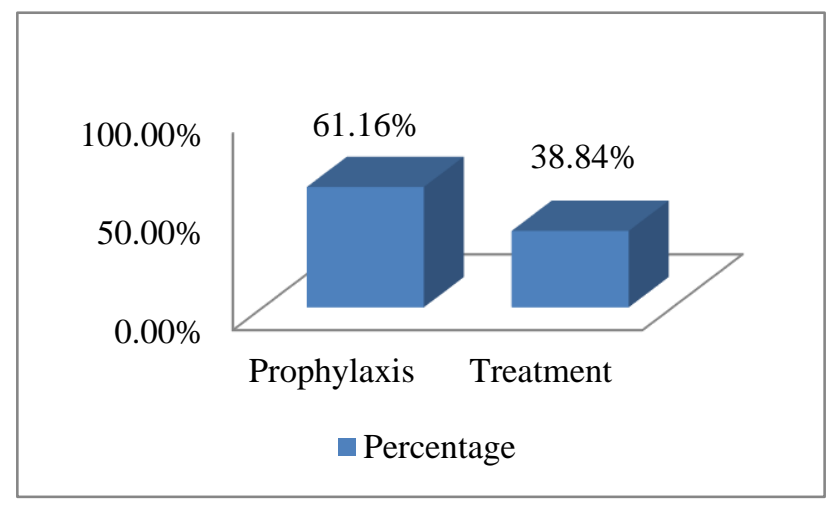

Figure 6: Indication for antimicrobials.

\section{Culture and sensitivity results}

From 55 patients' various specimens were sent for culture $\&$ sensitivity. In that 34 reports were positive for various organisms. 18 samples were sterile, 1 showed normal flora and 2 reports were not available.

Percentage of drugs prescribed by brand and generic name

Among the 816 antimicrobial agents prescribed, 74 were prescribed in generic names and remaining 742 were prescribed in brand names.

\section{Adverse drug reaction}

No adverse drug reaction to any antimicrobial agents prescribed was recorded in the case records studied

\section{DISCUSSION}

This observational study was carried out in the postoperative ward of general surgery for a period of one year. A total of 513 prescriptions were analyzed in the study period.

\section{Age distribution among post-operative patients}

In our study, most of the patients were seen in an increasing age group with the peak at fifth decade $(21.2 \%)$. Our study findings are similar to that of studies by Bhansali and Afzhal khan. A study by Neha et al showed that most of the patients were from third decade. This variation could be due to the small sample size and short duration of the study period. ${ }^{7}$

\section{Gender distribution}

This difference was statistically significant with $p$ value in our study most of the post-operative cases were males (56.34\%) similar to the study carried out by Rehan et al and Rana et al. ${ }^{8}$

\section{Diagnosis}

Most common diagnosis for surgery in our study was found to be hernia, abscess and appendicitis. Our study findings are similar to Bhansali. The reason for similarity is due to the common prevalence of these conditions which warrants surgery. ${ }^{8}$

\section{Pattern of antimicrobials prescribed}

Of the five hundred and thirteen patients studied no antimicrobials was prescribed to $29(5.7 \%)$ patients. Remaining 484 patients $(94.3 \%)$ were prescribed with at least one antimicrobial agent during their stay. One antimicrobial agent was prescribed for $162(33.5 \%)$ patients, two antimicrobial agents were prescribed for 190 
(39.3\%) patients, three antimicrobial agents were prescribed for $42(8.7 \%)$ patients and fixed dose combinations were prescribed for $90(18.5 \%)$ patients.

Studies by Amane et al in the past have mentioned that $52.59 \%$ received single drug, $15.55 \%$ received two or more drugs and $31.85 \%$ received FDC. ${ }^{9}$

In total of 484 patients, 816 antimicrobial agents were prescribed. 19 types of antimicrobial agents were utilized in this study. Average number of antimicrobial agents prescribed per patient is 1.72 . In contrast to the study findings by Chawda, we found that low number of antimicrobial agents were prescribed in our study. This will reduce the economic burden and also decreases the antimicrobial resistance. In our study, the most commonly used class of antimicrobial agent was the cephalosporin group $(52.32 \%)$ followed by metronidazole (34.38\%). Other group of antimicrobial agents were aminoglycosides $(6.58 \%)$, quinolones $(5.23 \%)$, penicillin $(0.9 \%)$, macrolides $(0.45 \%)$ and tetracycline $(0.14 \%)$. Our study findings are similar to that of studies by Patil et al and Khan et al. Cephalosporin has been proved as a very important class of a drug but its use beside the recommended protocol can lead to occurrence of resistance. So over usage of cephalosporins has to be avoided in the future.

Totally $135(27.89 \%)$ patients received drugs which are not included in the essential medicine list of WHO and 112 patients $(23.14 \%)$ received drugs which are not from national EML which is similar to that of Kamath. A survey led by Salman et al also supported our study findings in which only $62.2 \pm 18.1 \%$ of drugs were prescribed from the list. ${ }^{9}$

\section{Route of administration}

Antimicrobials were given intravenously in 438 (90\%) patients, orally in $42(9 \%)$ patients and both forms in 4 (1\%) patients. Chawda et al proved the same, where a greater number of antimicrobials was given through parentral route. As the post- operative patients are sick and as most of the antimicrobials are available in injection form and also due to more bioavailability with parenteral drugs, most of the antimicrobials were administered intravenously in this study.

\section{Duration}

The minimum duration of antimicrobial prescription was one day and the maximum duration was seventeen days. The average duration was found to be 4.09 days, which were higher when compared to Abula et al. Antimicrobials were stopped within 24 hours in 111 patients. Even though antimicrobials were stopped within 24 hours, no wound infection was reported in these cases. This was due to clean surgical procedure and minimal invasive surgeries performed.

\section{Indication for antimicrobials}

The common indication for the usage of antimicrobials was found to be prophylactic in $296(61.16 \%)$ patients and188 (38.84\%) patients received for treatment purposes mainly on empirical basis. The usage of antimicrobials for prophylaxis was found to be more eminent in our study similar to that of Bhabhor et al.

\section{Culture and sensitivity}

Specimens were sent for culture \& sensitivity from 55 patients. In that 34 reports were positive for organisms like E. coli, pseudomonas, $k$. Pneumonia and acinetobacter. Most of them showed sensitivity to commonly used antibiotics but few showed resistance to ampicillin, amoxicillin and cefotaxime. Remaining 18 samples were sterile, 1 showed normal flora and 2 reports were not available. Out of 484 patients' culture and sensitivity was done in $11.36 \%$ of cases only. Even though 3 patients showed resistance to cefotaxime they received the same drug post- operatively. This has to be avoided and efforts are being made to encourage the initiation of antimicrobials after obtaining culture and sensitivity reports.

\section{Drugs prescribed in brand and generic names}

In our study, most of the antimicrobials were prescribed by their brand name $(91 \%)$, a finding similar to that of Chawdha et al. But another study by Bhansali et al showed that equal number of drugs were prescribed in both generic and brand names. This may increase the economic burden of the patients as branded antimicrobials are costlier than the generic antimicrobials in the market. So special emphasis will be made to promote the prescription of drugs by generic index in the future. ${ }^{10}$

\section{Adverse drug reaction}

None of the patient's case records were documented with any adverse drug reaction due to the antimicrobials prescribed similar to the study carried out by Rehan.

\section{Other drugs}

Other drugs prescribed in our study were analgesics, antiulcer, anti- inflammatory, anti-emetics, laxatives, insulin and anti-hypertensives similar to that of Salman.

WHO prescribing indicators summary in our study were: Average No. of drugs per encounter- 9.54, Percentage of drugs prescribed by generic name- $9 \%$, Percentage of encounters with an antibiotic prescribed $94.3 \%$, percentage of encounters with an injection prescribed $93.14 \%$, percentage of drugs prescribed from essential drug list $82.97 \%$. 


\section{Limitations}

Cost analysis and defined daily dose were not done in the study, which is very much essential to observe the economic burden of the patients admitted to the postoperative ward, as it is an observational study, patient compliance could not be assessed, other post-operative wards were not included.

Above limitations can be satisfied in the future studies.

\section{CONCLUSION}

From our study we conclude that Cephalosporins are the most frequently used antimicrobial agent, in which cefotaxime was commonly prescribed. Antimicrobial prescription was considered to be rational regarding their dosage, route and the indication, but not supported by culture and sensitivity reports and duration. Reports from culture and sensitivity showed positive for organisms like E. coli, pseudomonas, $k$. Pneumonia and acinetobacter. Most of them showed sensitivity to commonly used antibiotics but few showed resistance to ampicillin, amoxicillin and cefotaxime. No adverse drug reaction to any antimicrobial prescribed in the post- operative ward has been noted in any of the patients. There was no change over in the antimicrobial agents during the course of treatment.

Rational prescribing of antibiotics would help avoid polypharmacy and prevent drug resistances. There is a clear need for the development of prescribing guidelines $\&$ educational initiatives to encourage the appropriate use of drugs in Surgery. Our study will help in promoting knowledge about essential medicine list among clinicians.

Funding: No funding sources

Conflict of interest: None declared

Ethical approval: The study was approved by the Institutional Ethics Committee

\section{REFERENCES}

1. Leaper DJ. Wound Infection. In: Russell RCG, William NS, Bulstrode CJK, eds. Bailey \& Love's
Short Practice of Surgery; 24th Edn. London: Arnold; 2004;118-32.

2. Nicholas RL. Wound Infection rates following clean operative procedures: Can assume them below (Editorial). Infect Cont Hosp Epidemiol. 1992;13:455.

3. Pea F, Viale P, Furlaunt MI. Antimicrobial agents in elective surgery: Prophylaxis or early therapy. J Chemother. 2003;15(1):3-11.

4. Patherick ES, Dalton JE. Methods for Identifying surgical wound infections after discharging from Hospital: a systematic review. BMC Infect Dis. 2006;6:170-8.

5. Skarzynska J, Cienciala A, Madry R, Barucha P, Kwasniak M. Hospital infection in general surgery wards. Przegl Epidemiol. 2000;54:299-304.

6. Kirkland KB, Briggs JP, Trivette SL, Wilkinson WE, Sexton DJ. The impact of surgical-site infections in the 1990s: Attributable mortality, excess length of hospitalization, and extra costs. Infect Control Hosp Epidemiol. 1999;20:725-30.

7. Esposito S, Leone S. Antimicrobial treatment for intensive care unit (ICU) infections, including the role of the infectious diseases specialist. Int $\mathrm{J}$ Antimicrob Agents. 2007;29:494-500.

8. Weber RJ, Kane SL, Oriolo VA, Saul M, Skledar SJ, Dasta JF. Impact of intensive care drug costs: A descriptive analysis, with recommendations for optimizing ICU pharmacotherapy. Crit Care Med. 2003;31:17-24.

9. Bhabhor P, Hotchandani H. An Antibacterial Drug Utilization Study at Surgical Units of Shree Sayaji General Hospital, Vadodara, Gujarat, India. The Internet J of Pharm. 2012;10(1).

10. Okeke IN, Lamikanra A, Edelman R. Socioeconomic and behavioral factors leading to acquired bacterial resistance to antibiotics in developing countries. Emerg Infect Dis. 1999;5:18-27.

Cite this article as: Ramalingam V, Johan PJ. A prospective observational study on prescribing pattern of antimicrobial agents in the post-operative general surgery ward of a tertiary care teaching hospital. Int J Basic Clin Pharmacol. 2020;9:1513-9. 